\title{
On a Control Problem Containing Support Functions
}

\author{
I. Husain', A. Ahmed2, Abdul Raoof Shah² \\ ${ }^{1}$ Department of Mathematics, Jaypee University of Engineering and Technology, Guna, India \\ ${ }^{2}$ Department of Statistics, University of Kashmir, Srinagar, India \\ Email: ihusain11@yahoo.com
}

Received 14 June 2014; revised 15 July 2014; accepted 10 August 2014

Copyright (C) 2014 by authors and Scientific Research Publishing Inc.

This work is licensed under the Creative Commons Attribution International License (CC BY). http://creativecommons.org/licenses/by/4.0/

(c) (i) Open Access

\begin{abstract}
A control problem containing support functions in the integrand of the objective of the functional as well as in the inequality constraint function is considered. For this problem, Fritz John and Karush-Kuhn-Tucker type necessary optimality conditions are derived. Using Karush-Kuhn-Tucker type optimality conditions, Wolfe type dual is formulated and usual duality theorems are established under generalized convexity conditions. Special cases are generated. It is also shown that our duality results have linkage with those of nonlinear programming problems involving support functions.
\end{abstract}

\section{Keywords}

Control Problem, Support Function, Optimality Conditions, Generalized Convexity, Wolfe Type Duality, Nonlinear Programming Problem

\section{Introduction}

Optimal control theory, which is an extension of calculus of variations is a mathematical optimization method for deriving control policies. In essence, an optimal control is set of differential equations describing the path of the control variables that minimize the cost functional. Mond and Hanson [1] were the first to formulate a control problem as a mathematical programming problem and studied Wolfe type duality for the same under convexity of the function involved in the formulation. Subsequently a number of duality results for a control problem involving differentiable functions were obtained, for example, in the references [2]-[5]. There exist applications of optimal control with nondifferentiable terms which appear in the problem of friction. This motivated Chandra et al. [2] to study optimality and duality for a class of nondifferentiable control problem containing the square root of certain quadratic form in the integrand of the objective functional. The popularity of this type of 
mathematical programming problem seems to originate from the fact that even though the objective functions and/or constraint functions are nonsmooth, a simple representation for the dual may be found. Non smooth mathematical programming theory deals with much more general functions by means of generalized subdifferential [6] and quasidifferential [7]. However, the square root of a positive semidefinite quadratic form and support function are of the few cases of a nondifferentiable function for which subdifferentials can explicitly be written.

In this research we introduce a control problem with a support function in the integrand of the objective functional and each inequality constraint function. Optimality conditions for this nondifferentiable control problem are derived and Wolfe type duality is investigated under pseudoconvexity. Special cases are generated. The linkage between our results and those of nonlinear programming problem containing support function is also indicated.

\section{Control Problem and Preliminaries}

We introduce the following control problem involving support functions:

(CP): Minimize: $\int_{I}\{f(t, x, u)+s(u(t) \mid K)\} \mathrm{d} t$.

Subject to

$$
\begin{gathered}
x(a)=\alpha, \quad \alpha(b)=\beta \\
\dot{x}(t)=h(t, x, u), \quad t \in I \\
g^{j}(t, x, u)+s\left(x(t) \mid C^{j}\right) \leq 0, \quad t \in I, \quad j=1,2, \cdots, m
\end{gathered}
$$

where

1) $x: I \rightarrow R^{n}$ is a differentiable state vector function with its derivative $\dot{x}$ and $u: I \rightarrow R^{m}$ is a smooth control vector function.

2) $R^{n}$ denotes an $n$-dimensional Euclidean space and $I=[a, b]$ is a real interval, and

3) $f: I \times R^{n} \times R^{m} \rightarrow R, \quad g^{j}: I \times R^{n} \times R^{m} \rightarrow R, j=1,2, \cdots, m$ and $h: I \times R^{n} \times R^{m} \rightarrow R^{n}$ are continuously differentiable.

4) $s(x(t) \mid K)$ and $s\left(x(t) \mid C^{j}\right), j=1,2, \cdots, m$ are the support function of the compact set $K$ and $C^{j}(j=1,2, \cdots, m)$ respectively.

Denote the partial derivatives of $f$ by $f_{t}, f_{x}$ and $f_{y}$,

$$
f_{t}=\frac{\partial f}{\partial t}, f_{x}=\left[\frac{\partial f}{\partial x^{1}}, \frac{\partial f}{\partial x^{2}}, \cdots, \frac{\partial f}{\partial x^{n}}\right], f_{u}=\left[\frac{\partial f}{\partial u^{1}}, \frac{\partial f}{\partial u^{2}}, \cdots, \frac{\partial f}{\partial u^{m}}\right],
$$

where superscript denote the vector components. Further $X$ represents the space of continuously differentiable state functions $x: I \rightarrow R^{n}$ such that $x(a)=0$ and $x(b)=0$ and is equipped with the norm $\|x\|=\|x\|_{\infty}+\left\|D_{x}\right\|_{\infty}$, and $U$, the space of piecewise continuous control vector functions $u: I \rightarrow R^{m}$ having the uniform norm $\|\cdot\|_{\infty}$. The differential Equation (2) with initial conditions expressed as $x(t)=x(a)+\int_{a}^{b} h(s, x(s), y(s)) \mathrm{d} s \quad t \in I$ may be written as $H_{x}=H(x, y)$, where $H: X \times U \rightarrow C\left(I, R^{n}\right), C\left(I, R^{n}\right)$ being the space of continuous function from $I$ to $R^{n}$ defined as $H(x, y)(t)=h(t, x(t), y(t))$. In the derivation of these optimality condition, some constraint qualification to make the equality constraint locally solvable [2] is needed for this and hence, the Fréchet derivative of $D_{x}-H(x, u)=Q(x, u)$, (say) with respect to $(x, u)$, namely

$Q^{\prime}=Q^{\prime}(x, u)=\left[D-H_{x}(x, u),-H_{u}(x, u)\right]$ are required to be subjective. We review some well known facts about a support function for easy reference. Let $\Gamma$ be a compact convex set in $R^{n}$. Then the support function of $\Gamma$ denoted by $s(x(t) \mid \Gamma)$ is defined as, $s(x(t) \mid \Gamma)=\operatorname{Max}\left\{x(t)^{\mathrm{T}} v(t): v(t) \in \Gamma, t \in I\right\}$.

A support function, being convex and everywhere finite, has a subdifferential in the sense of convex analysis, that is, there exists $z$ such that $s(y(t) \mid \Gamma) \geq s(x(t) \mid \Gamma)+z(t)^{\mathrm{T}}(y(t)-x(t))$ for all $x$. 
As in [8] the subdifferential of $s(x(t) \mid \Gamma)$ is given by $s(x(t) \mid \Gamma)=\left\{z(t) \in \Gamma: z(t)^{\mathrm{T}} x(t)=s(x(t) \mid \Gamma)\right\}$. Let $N_{\Gamma}(x(t))$ be normal cone at a point $x(t) \in \Gamma$. Then $y(t) \in N_{\Gamma}(x)$ if and only if $s(y(t) \mid \Gamma)=x(t)^{\mathrm{T}} y(t)$ or equivalently, $x(t)$ is in the subdifferential of $s$ at $y(t)$.

\section{Optimality Conditions}

In this section, we derive necessary optimality conditions of both Fritz John and Karush-Kuhn-Tucker type for the control problem (CP) stated in the preceding section.

Theorem 1. (Fritz John Conditions): If $(\bar{x}, \bar{u})$ is an optimal solution of (CP) and the Fréchet derivative $Q^{\prime}$ is surjective, then there exist Langrange multipliers $\alpha \in R$ and piecewise smooth

$\lambda: I \rightarrow R^{m}, \mu: I \rightarrow R^{n}, z: I \rightarrow R^{m}$ and $\omega^{j}: I \rightarrow R^{n}$ such that

$$
\begin{gathered}
\alpha f_{x}(t, \bar{x}, \bar{u})+\sum_{j=1}^{m} \lambda^{j}(t)\left(g_{x}^{j}(t, \bar{x}, \bar{u})+\omega^{j}(t)\right)+\mu(t)^{\mathrm{T}} h_{x}(t, \bar{x}, \bar{u})+\dot{\mu}(t)=0, \quad t \in I \\
\alpha f_{u}(t, \bar{x}, \bar{u})+\lambda^{\mathrm{T}}(t) g_{u}(t, \bar{x}, \bar{u})+\mu(t)^{\mathrm{T}} h_{u}(t, \bar{x}, \bar{u})=0, \quad t \in I \\
\sum_{j=1}^{m} \lambda^{j}(t)\left(g_{x}^{j}(t, \bar{x}, \bar{u})+x^{\mathrm{T}}(t) \omega^{j}(t)\right)=0, \quad t \in I \\
\mu^{\mathrm{T}}(t) z(t)=s(x(t) \mid K), \quad t \in I \\
x(t) \omega^{j}(t)=s\left(x(t) \mid C^{j}\right), \quad j=1,2, \cdots, m, \quad t \in I \\
z(t) \in K, \omega^{j}(t) \in C^{j}, \quad j=1,2, \cdots, m \\
(\alpha, \lambda(t)) \geq 0, \quad t \in I \\
(\alpha, \lambda(t), \mu(t)) \neq 0, \quad t \in I .
\end{gathered}
$$

Proof: The problem (CP) may be expressed in its abstract version as

(ECD): $\underset{(x, u)}{\operatorname{Minimize}} \phi(x, u)=F(x, u)+\psi(x)$

subject to

$$
\begin{aligned}
& D_{x}=H(x, u) \\
& G(x, u) \in S
\end{aligned}
$$

where $F(x, u)=\int_{I} f(t, x, u), \psi(u)=\int_{I} s(u(t) \mid K) \mathrm{d} t, \quad G:(X \times U) \rightarrow C\left(I, R^{m}\right) \quad$ is given by $\quad($ for all $t \in I)$, $G^{j}(x, u)(t)=g^{j}(t, x, u)+s\left(x(t) \mid C^{j}\right), j=1,2, \cdots, m$ and $S=C_{+}\left(I, R^{m}\right)$; the nonnegative orthant of $C\left(I, R^{n}\right)$.

By the result of [9] it follows that there exist Langrange multipliers $\alpha \in R, \rho \in S^{*}$ (the dual of $S$ ) and $v$ in the dual space of $C_{+}\left(I, R^{m}\right)$ satisfying

$$
\begin{gathered}
0 \in \alpha \partial \phi(\bar{x}, \bar{u})+\partial\left(\rho^{\mathrm{T}} G(\bar{x}, \bar{u})\right)+\partial v^{\mathrm{T}}(D \bar{x}-H(\bar{x}, \bar{u})) \\
\rho^{\mathrm{T}} G(\bar{x}, \bar{u})=0, \\
(\alpha, \rho) \geq 0 \\
(\alpha, \rho, v) \neq 0 .
\end{gathered}
$$

The condition (12) reduces to

$$
0 \in\left\{\alpha F_{x}(x, u)\right\}+\left\{\rho^{\mathrm{T}} G_{x}^{1}(x, u)\right\}+\partial \rho^{\mathrm{T}} G_{x}^{2}(x, u)+\left\{v^{\mathrm{T}}\left(D-H_{x}(\bar{x}, \bar{u})\right)\right\}
$$




$$
0 \in\left\{\alpha F_{u}(\bar{x}, \bar{u})\right\}+\partial(\alpha \psi(u))+\rho^{\mathrm{T}} G_{u}^{1}(\bar{x}, \bar{u})+\left\{v^{\mathrm{T}} H_{u}(\bar{x}, \bar{u})\right\} .
$$

Since $f$ is continuously differentiable function of $x$ and $u, F(x, u)$ is Fréchet differentiable with respect to $(x, u)$. The partial derivatives of $F$ with respect to $\bar{x}$ and $u$, denoted by $F_{x}(\bar{x}, \bar{u})$ and $F_{u}(\bar{x}, \bar{u})$ respectively, are given by

$$
\begin{gathered}
(\forall p \in X) F_{x}(x, u) p=\int_{I} f_{x}(t, \bar{x}, \bar{u}) p(t)+f_{\dot{x}}(t, \bar{x}, \bar{u}) p(t) \mathrm{d} t \\
(\forall q \in X) F_{u}(x, u) q=\int_{I} f_{u}(t, x, u) q(t) \mathrm{d} t .
\end{gathered}
$$

Similar results for $g$ and $h$ as for $f$ can be given. Assume now subject to later validation that, $\rho \in S^{*}$ can be represented by measurable function $\lambda: I \rightarrow R^{m}$ with $\lambda=\left(\lambda^{1}, \lambda^{2}, \cdots, \lambda^{m}\right)$ satisfying

$$
\left(\forall \xi \in C\left(I, R^{m}\right)\right),\langle\rho, \xi\rangle=\int_{I} \lambda(t)^{\mathrm{T}} \xi(t) \mathrm{d} t .
$$

Define the convex function $\eta_{t}: R^{n} \rightarrow R$ by $\eta_{t}(u)=s(u(t) \mid K)$. From [2] its subdifferential,

$$
\partial \eta_{t}(v)=\left\{z \mid z \in K, \eta_{t}(u)=u^{\mathrm{T}} z\right\}
$$

Now $Q(x)=\int_{I} \eta_{t}(u) \mathrm{d} t$. From ([6], Theorem 3), we have

$$
y \in \partial Q(\bar{u}) \Leftrightarrow\left\{(\forall t \in I), \sigma(t) \in \partial \eta_{t}(\bar{u}),\langle y, v\rangle=\int_{I} \sigma(t)^{\mathrm{T}} u(t) \mathrm{d} t\right\}
$$

with $\sigma: I \rightarrow R^{n}$ measurable, namely $\sigma(t)^{\mathrm{T}}=z(t), t \in I$ from (16).

Let $\xi(t, x(t))=s\left(x(t) \mid C^{(\cdot)}\right)$, where $s\left(x(t) \mid C^{(\cdot)}\right)$ denotes the vector support function whose $j^{\text {th }}$ component is $s\left(x(t) \mid C^{(j)}\right)$. Then

$$
\rho^{\mathrm{T}} \xi(., x)=\int_{I} \lambda(t)^{\mathrm{T}} \xi(t, x(t)) \mathrm{d} t .
$$

Denoted by $\partial_{c}$ the Clarke generalized gradient [6] with respect to $x$. Then

$$
\begin{aligned}
\partial_{c}\left(\lambda(t)^{\mathrm{T}} \xi(t, x(t))\right) \subset \sum_{j=1}^{m} \partial_{c}\left(\lambda^{j}(t)\left(\xi^{j}(t, x(t))\right)\right) & =\sum_{j=1}^{m}\left|\lambda^{j}(t)\right| \partial_{c}\left(\operatorname{sgn}\left(\lambda^{j}(t)\right) \xi^{j}(t, x(t))\right) \\
& =\sum_{j=1}^{m}\left|\lambda^{j}(t)\right| \operatorname{sgn}\left(\lambda^{j}(t)\right) \partial_{c}\left(\xi^{j}(t, x(t))\right) .
\end{aligned}
$$

The above is possible by using the representation of $\partial_{c}($.$) as the convex hull of limit of points of gradients$ at smooth points near $x$. Here $\sum$ denotes the algebraic sum of sets. Since $\xi^{j}(t,)=.s\left(() \mid. C^{j}\right)$ is convex, we have for each $j \in\{1,2, \cdots, m\}$,

$$
\partial_{c}(.) \xi^{j}(t, x(t))=\partial_{c} \xi^{j}(t, x(t))=\left\{\omega^{j}(t) \mid \omega^{j}(t) \in C^{j}, \xi^{j}(t, x(t))=\bar{x}(t) \omega^{j}(t), t \in I\right\} .
$$

From [10], it implies that $q \in \partial\left(\rho^{\mathrm{T}} \xi\right)(., x)$ if and only if these exists a measurable function $\rho: I \rightarrow R^{m}$ such that

$$
(\forall t \in I), q(t) \in \partial_{c} \xi(t, x(t)) ; \quad(\forall p \in X),\langle q, p(t)\rangle=\int_{I} \lambda(t)^{\mathrm{T}} \rho(t) p(t) \mathrm{d} t .
$$

Now

$$
\begin{aligned}
\partial_{x}\left(\rho^{\mathrm{T}} G\right)(\bar{x}, \bar{u}) & =\left\{\theta^{\mathrm{T}} p \mid \theta \in \partial\left(\rho^{\mathrm{T}} G\right)(\bar{x})\right\} \\
& =\sum_{j=1}^{m} \int_{I} \lambda^{j}(t)\left[g_{x}^{j}(t, \bar{x}(t), \dot{\bar{x}}(t)) p(t)+g_{\dot{x}}^{j}(t, \bar{x}(t), \dot{\bar{x}}(t)) \dot{p}(t)+\omega^{j}(t)^{\mathrm{T}} p(t)\right] \mathrm{d} t .
\end{aligned}
$$

Consider, 


$$
\begin{aligned}
v\left(H_{x}-D\right) p & =\int_{I}\left(\mu(t)^{\mathrm{T}} h_{x} p-\mu(t) \dot{p}\right) \mathrm{d} t=\int_{I} \mu(t) h_{x} p \mathrm{~d} t-\left.\mu(t) p\right|_{a} ^{b}+\int_{I} \dot{\mu}(t) p \mathrm{~d} t \\
& =\int_{I}\left(\mu(t)^{\mathrm{T}} h_{x}+\dot{\mu}(t)\right) p \mathrm{~d} t \quad(\operatorname{using} \mu(a)=0=\mu(b)) .
\end{aligned}
$$

Using (18), (25), (26), we have

$$
\int_{I}\left[\alpha f(t, \bar{x}, \bar{u})+\sum_{j=1}^{m} \lambda^{j}(t)\left(g_{x}^{j}(t, \bar{x}, \bar{u})+\omega^{j}(t)\right)+\mu(t)^{\mathrm{T}} h_{x}(t, \bar{x}, \bar{u})+\dot{\mu}(t)\right] p(t) \mathrm{d} t=0 .
$$

Since the integral values for any $v \in X$, by Lemma 2 ([11], p. 500), it follows that

$$
\alpha f_{x}(t, \bar{x}, \bar{u})+\sum_{j=1}^{m} \lambda^{j}(t)\left(g_{x}^{j}(t, \bar{x}, \bar{u})+\omega^{j}(t)\right)+\mu(t)^{\mathrm{T}} h_{x}(t, \bar{x}, \bar{u})+\dot{\mu}(t)=0 \quad t \in I .
$$

The cited lemma assumes that the expression in the square bracket of (27) is piecewise continuous, but this readily extends to measurable. This validates (4). On the basis of analysis needed to validate (28), we can easily establish

$$
f_{x}(t, \bar{x}, \bar{u})+z(t)+\lambda(t)^{\mathrm{T}} g_{u}(t, \bar{x}, \bar{u})+\mu(t)^{\mathrm{T}} h_{u}(t)=0, \quad t \in I .
$$

Also $\sum_{j=1}^{m} \bar{\rho}^{j} G^{j}(\bar{x})=0$ along with $\bar{x}(t)^{\mathrm{T}} \bar{\omega}^{j}(t)=\left(\bar{x}(t) \mid C^{j}\right)$ of (24) yields

$$
\sum_{j=1}^{m} \int_{I} \bar{\lambda}^{j}(t)\left[g^{j}(t, \bar{x}(t), \dot{\bar{x}}(t))+\bar{x}(t)^{\mathrm{T}} \omega^{j}(t)\right] v(t) \mathrm{d} t=0 .
$$

By the application of the above-cited lemma, this gives (6) i.e.

$$
\sum_{j=1}^{m} \lambda^{j}(t)\left[g^{j}(t, \bar{x}(t), \dot{\bar{x}}(t))+\bar{x}(t)^{\mathrm{T}} \bar{\omega}^{j}(t)\right]=0, \quad t \in I .
$$

The remaining proof of the theorem easily follow on the lines of the proof of Theorem 4.1 of [2].

Hence the above analysis established the theorem fully.

Chandra et al. [2] pointed out if the optimal solution for (CP) is normal, then the Fritz John type optimal conditions reduce to the following Karush-Kuhn-Tucker optimal conditions:

Theorem 2: If $(\bar{x}, \bar{u})$ is an optimal solution and is normal and $Q^{\prime}$ is surjective, there exist piecewise smooth $\lambda^{j}: I \rightarrow R^{m}, j=1,2, \cdots, m$ with $\lambda^{T}=\left(\lambda_{1}, \lambda_{2}, \cdots, \lambda_{m}\right), \quad \mu: I \rightarrow R^{n}, \quad z: I \rightarrow R^{n}$ and $\omega^{j}: I \rightarrow R^{n}$, $j=1,2, \cdots, m$.

Such that

$$
\begin{gathered}
f_{x}(t, \bar{x}, \bar{u})+\sum_{j=1}^{m} \lambda^{j}(t)\left(g_{x}^{j}(t, \bar{x}, \bar{u})+\omega^{j}(t)\right)+\mu(t)^{\mathrm{T}} h_{x}(t, \bar{x}, \bar{u})=\dot{\mu}(t) \\
f_{u}(t, \bar{x}, \bar{u})+\lambda^{\mathrm{T}}(t) g_{u}(t, \bar{x}, \bar{u})+\mu(t)^{\mathrm{T}} h_{u}(t, \bar{x}, \bar{u})=0, \quad t \in I \\
\sum_{j=1}^{m} \lambda^{j}(t)\left(g_{x}^{j}(t, \bar{x}, \bar{u})+x^{\mathrm{T}}(t) \omega^{j}(t)\right)=0, \quad t \in I \\
u(t)^{\mathrm{T}} z(t)=s(x(t) \mid K) \\
x(t)^{\mathrm{T}} \omega^{j}(t)=s\left(x(t) \mid C^{j}\right), \quad j=1,2, \cdots, m \\
\lambda^{j}(t) \geq 0, \quad t \in I, \quad j=1,2, \cdots, m \\
z(t) \in K, \omega^{j}(t) \in C^{j}, \quad j=1,2, \cdots, m .
\end{gathered}
$$




\section{Wolfe Type Duality}

We propose the following dual as the Wolfe type dual and validate duality results amongst (CP) and (WCD).

(WCD): Maximize

$$
\int_{I}\left\{f(t, x, u)+u(t)^{\mathrm{T}} z(t)+\sum_{j=1}^{m} \lambda^{i}(t)\left(g^{j}(t, x, u)+x(t)^{\mathrm{T}} \omega^{j}(t)\right)+\mu(t)^{\mathrm{T}}(h(t, x, u)-\dot{x}(t))\right\} \mathrm{d} t
$$

subject to

$$
\begin{gathered}
x(a)=0, \quad \alpha(b)=0 \\
f_{x}(t, x, u)+\sum_{j=1}^{m} \lambda^{i}(t)\left(g^{j}(t, x, u)+\omega^{j}(t)\right)+\mu(t)^{\mathrm{T}} h_{x}(t, x, u)+\dot{\mu}(t)=0, \quad t \in I \\
f_{u}(t, x, u)+\lambda^{\mathrm{T}}(t) g(t, x, u)+\mu(t)^{\mathrm{T}} h_{u}(t, x, u)=0, \quad t \in I \\
\lambda^{i}(t) \geq 0, \quad t \in I, \quad i=1,2, \cdots, m \\
z(t) \in K, \omega^{j}(t) \in C^{j}, \quad j=1,2, \cdots, m .
\end{gathered}
$$

Theorem 3 (Weak Duality): Assume that

1) $(\bar{x}, \bar{u})$ is feasibility for (CP)

2) $\left(x, u, \lambda, u, z, \omega^{1}, \omega^{2}, \cdots, \omega^{m}\right)$ is feasible for (CD) and

3) for all feasible, $\left(\bar{x}, \bar{u}, x, u, \lambda, u, z, \omega^{1}, \omega^{2}, \cdots, \omega^{m}\right)$

$$
\int_{I}\left\{f+\sum_{j=1}^{m} \lambda^{i}(t)\left(g^{j}(., .)+(.)^{\mathrm{T}} \omega^{j}(.)\right)+\mu^{\mathrm{T}}(t)(h-\dot{x}(t))\right\} \mathrm{d} t
$$

is pseudo convex in $(x, u)$ for all $z(t) \in R^{m}$ and $\omega^{j}(t) \in R^{n}, j=1,2, \cdots, m$.

Then

$$
\inf (\mathrm{CP}) \geq \operatorname{Sup}(\mathrm{WCD}) \text {. }
$$

Proof: Combining (37) and (38), we have

$\int_{I}\left[(\bar{x}-x)^{\mathrm{T}}\left\{f_{x}+\sum_{j=1}^{m} \lambda^{j}(t)\left(g_{x}^{j}+\omega^{j}(t)\right)+\mu(t)^{\mathrm{T}}\left(h_{x}-\dot{\mu}(t)\right)\right\}+(\bar{u}-u)\left\{f_{u}+z(t)+\lambda(t)^{\mathrm{T}} g_{u}+\mu(t)^{\mathrm{T}} h_{u}\right\}\right] \mathrm{d} t=0$.

By the pseudoconvexity hypothesis 3), this yields

$$
\begin{aligned}
& \int_{I}\left\{f(t, \bar{x}, \bar{u})+\bar{x}(t)^{\mathrm{T}} z(t)+\sum_{j=1}^{m} \lambda^{j}(t)\left(g^{j}(t, x, \bar{u})\right)+\bar{x}(t)^{\mathrm{T}} \omega^{j}(t)+\mu(t)(h(t, \bar{x}, \bar{u})-\dot{\bar{x}}(t))\right\} \mathrm{d} t \\
& \quad \geq \int_{I}\left\{f(t, x, u)+x(t)^{\mathrm{T}} z(t)+\sum_{j=1}^{m} \lambda^{j}(t)\left(g^{j}(t, x, u)\right)+x(t)^{\mathrm{T}} \omega^{j}(t)+\mu(t)(h(t, x, u)-\dot{x}(t))\right\} \mathrm{d} t .
\end{aligned}
$$

Since $(\bar{x}, \bar{u})$ is feasible for (CP), we have

$$
h(t, \bar{x}, \bar{u})=\dot{\bar{x}}, \quad t \in I
$$

implying

$$
\mu(t)^{\mathrm{T}}(h(t, \bar{x}, \bar{u})-\dot{\bar{x}}(t))=0, \quad t \in I
$$

and

$$
g^{i}(t, \bar{x}, \bar{u})+s\left(\bar{x}(t) \mid C^{j}\right) \leq 0, \quad t \in I
$$

implying 


$$
\Rightarrow \sum \lambda(t)\left(g^{j}(t, \bar{x}, \dot{u})+s\left(x(t) \mid C^{j}\right)\right) \leq 0, t \in I, \quad t \in I .
$$

Since $\bar{x}(t)^{\mathrm{T}} \omega^{j}(t) \leq s\left(x(t) \mid C^{j}\right), \quad t \in I$, we have

$$
\sum_{j=1}^{m} \lambda^{j}(t)\left(g^{j}(t, \bar{x}, \bar{u})+x(t)^{\mathrm{T}} \omega^{j}(t)\right) \leq \sum_{j=1}^{m} \lambda^{j}(t)\left(g^{j}(t, \bar{x}, \bar{u})\right)+s\left(\bar{x}(t) \mid C^{j}\right) \leq 0, \quad t \in I .
$$

From (41), we have

$$
\begin{aligned}
& \int_{I}\left\{f(t, \bar{x}, \bar{u})+s(\bar{u}(t) \mid K)+\sum_{j=1}^{m} \lambda^{j}(t)\left(g^{j}(t, \bar{x}, \bar{u})\right)+s\left(\bar{x}(t) \mid C^{j}\right)+\bar{\mu}(t)(h(t, \bar{x}, \bar{u})-\dot{\bar{x}})\right\} \mathrm{d} t \\
& \quad \geq \int_{I}\left\{f(t, x, u)+\sum_{j=1}^{m} \lambda^{j}(t)\left(g^{j}(t, x, u)\right)+x(t)^{\mathrm{T}} \omega^{j}(t)+\mu(t)(h(t, x, u)-\dot{x}(t))\right\} \mathrm{d} t .
\end{aligned}
$$

This implies

$$
\int_{I}\{f(t, \bar{x}, \bar{u})+S(\bar{x}(t) \mid K)\} \mathrm{d} t \geq \int_{I}\left\{f(t, x, u)+\sum_{j=1}^{m} \lambda^{j}(t)\left(g^{j}(t, x, u)\right)+x(t)^{\mathrm{T}} \omega^{j}(t)+\mu(t)(h(t, x, u)-\dot{x}(t))\right\} \mathrm{d} t .
$$

That is,

$$
\inf (\mathrm{CP}) \geq \operatorname{Sup}(\mathrm{CD}) \text {. }
$$

Theorem 4 (Strong duality): If $(\bar{x}, \bar{u})$ is an optimal solution of (CP) and is normal, there exist piecewise smooth $\lambda: I \rightarrow R^{m}$ where $\left(\lambda=\lambda_{1}, \lambda_{2}, \cdots, \lambda_{m}\right), \quad z: I \rightarrow R^{n}, \mu: I \rightarrow R^{n}$ and $\omega^{j}: I \rightarrow R^{n}, \quad(j=1,2, \cdots, m$. $)$ such that $\left(\bar{x}, \bar{u}, z, \bar{\lambda}, \bar{\mu}, \bar{\omega}^{1}, \cdots, \bar{\omega}^{m}\right)$ is feasible for (WCD) and the optimal values of the problem (CP) and (WCD) are equal. If also the hypotheses of Theorem1 hold, then $\left(\bar{x}, \bar{u}, z, \bar{\lambda}, \bar{\mu}, \bar{\omega}^{1}, \cdots, \bar{\omega}^{m}\right)$ is an optimal solution of the problem (WCD).

Proof: Since $(\bar{x}, \bar{u})$ is an optimal solution of (CP) and is normal, by Theorem 1, it implies that there exist piecewise smooth $\lambda^{j}: I \rightarrow R, \quad j=1,2, \cdots, m, \quad u: I \rightarrow R^{n}, \quad z: I \rightarrow R^{n}$ and $\omega^{j}: I \rightarrow R^{n} \quad(j=1,2, \cdots, m$. $)$ such that conditions (4)-(10) of the theorem are satisfied. The conditions (4)-(6) together with (9) and (10) imply the feasibility of $\left(\bar{x}, \bar{u}, z, \bar{\lambda}, \bar{\mu}, \bar{\omega}^{1}, \cdots, \bar{\omega}^{m}\right)$ for (WCD). The condition (6)-(8) yield the equality of objective functionals of the two problem. In view of this equality and the hypotheses of Theorem 3, the optimality of $\left(\bar{x}, \bar{u}, \bar{z}, \bar{\lambda}, \bar{\mu}, \bar{\omega}^{1}, \cdots, \bar{\omega}^{m}\right)$ for (WCD) is obtained.

Theorem 5: (Strict Converse Duality): Assume

$\left(\mathbf{H}_{\mathbf{1}}\right):(\bar{x}, \bar{u})$ is an optimal solution and is normal;

$\left(\mathbf{H}_{2}\right):\left(\hat{x}, \hat{u}, \hat{\lambda}, \hat{z}, \hat{\omega}^{1}, \cdots, \hat{\omega}^{m}, \hat{\mu}\right)$ is an optimal solution;

$\left(\mathbf{H}_{3}\right): \int_{I}\left[\sum_{j=1}^{m} \hat{\lambda}^{j}(t)\left(g^{j}(t, . .)+.(.)^{\mathrm{T}} \omega^{j}(t)\right)+\hat{u}(t)(h(t, . .)=.\hat{x}(t))\right] \mathrm{d} t$ is strictly pseudo convex.

then $(\bar{x}, \bar{u})=(\hat{x}, \hat{u})$, i.e. $\hat{u}$ is an optimal solution of $(\mathrm{CP})$.

Proof: Assume that $(\bar{x}, \bar{u}) \neq(\hat{x}, \hat{u})$. By Theorem 4, there exist piecewise smooth $\bar{\lambda}: I \rightarrow R^{m}$ with, $\bar{\lambda}(t)=\left(\lambda^{1}(t), \cdots, \lambda^{m}(t)\right), \bar{z}(t) \in K, \quad t \in I, \quad \bar{\mu}(t) \in R^{n}$ and $\bar{\omega}^{j}=\bar{\omega}^{j}(t), \quad t \in I, \quad j=1,2, \cdots, m$ such that $\left(\bar{x}, \bar{u}, \bar{\lambda}, \bar{u}, \bar{z}, \bar{\omega}^{1}, \cdots, \bar{\omega}^{m}\right)$ is an optimal to (CD) and

$$
\begin{aligned}
& \int_{I} \sum_{j=1}^{m} \hat{\lambda}^{i}(t)\left(g^{j}(t, \bar{x}, \bar{u})+\bar{x}(t)^{\mathrm{T}} \bar{\omega}^{i}(t)+\bar{\mu}(t)^{\mathrm{T}}(h(t, \bar{x}, \bar{u})-\dot{\bar{x}}(t))\right) \mathrm{d} t \\
& \quad=\int_{I} \sum_{j=1}^{m} \hat{\lambda}^{i}(t)\left(g^{j}(t, \hat{x}, \hat{u})+\hat{x}(t)^{\mathrm{T}} \hat{\omega}^{i}(t)+\hat{\mu}(t)^{\mathrm{T}}(h(t, \hat{x}, \hat{u})-\dot{\hat{x}}(t))\right) \mathrm{d} t .
\end{aligned}
$$

From the feasibility of $\left(\hat{x}, \hat{u}, \hat{\lambda}, \hat{u}, \hat{z}, \hat{\omega}^{1}, \cdots, \hat{\omega}^{m}\right)$ for (WCD), we have 


$$
\begin{array}{r}
\int_{I}\left\{(\bar{x}-\hat{x})^{\mathrm{T}}\left(f_{x}(t, \hat{x}, \hat{u})+\sum_{j=1}^{m} \hat{\lambda}^{j}(t)\left(g_{x}^{i}(t, \hat{x}, \hat{u})+\hat{\omega}^{j}(t)\right)+\mu(t)^{\mathrm{T}} h_{x}(t, \hat{x}, \hat{u})-\hat{\mu}(t)\right)\right. \\
\left.+(\bar{u}-\hat{u})\left(f_{u}(t, \hat{x}, \hat{u})+\hat{\mu}(t) \hat{z}(t)+\hat{\lambda}(t)^{\mathrm{T}} g_{u}(t, \hat{x}, \hat{u})+\hat{\mu}(t)^{\mathrm{T}} h_{u}(t, \hat{x}, \hat{u})\right)\right\} \mathrm{d} t=0 .
\end{array}
$$

This by strict pseudoconvexity hypothesis $\left(\mathrm{H}_{3}\right)$ yields,

$$
\begin{aligned}
& \int_{I}\left[f(t, \bar{x}, \bar{u})+\bar{u}(t)^{\mathrm{T}} \hat{z}(t)+\sum_{j=1}^{m} \hat{\lambda}^{j}(t)\left(g^{j}(t, \bar{x}, \bar{u})+\bar{x}(t) \hat{\omega}^{j}(t)\right)+\hat{\mu}(t)^{\mathrm{T}}(h(t, \bar{x}, \bar{u})-\dot{\bar{x}}(t))\right] \mathrm{d} t \\
& \quad \geq \int_{I}\left[f(t, \hat{x}, \hat{u})+\hat{u}(t)^{\mathrm{T}} \hat{z}(t)+\sum_{j=1}^{m} \hat{\lambda}^{j}(t)\left(g^{j}(t, \hat{x}, \hat{u})+\hat{x}(t) \hat{\omega}^{j}(t)\right)+\hat{\mu}(t)^{\mathrm{T}}(h(t, \hat{x}, \hat{u})-\dot{\hat{x}}(t))\right] \mathrm{d} t \\
& \quad=\int_{I}\left[f(t, \bar{x}, \bar{u})+\bar{u}(t)^{\mathrm{T}} \bar{z}(t)+\sum_{j=1}^{m} \hat{\lambda}^{j}(t)\left(g^{j}(t, \bar{x}, \bar{u})+\bar{x}(t)^{\mathrm{T}} \bar{\omega}^{j}(t)\right)+\bar{\mu}(t)^{\mathrm{T}}(h(t, \bar{x}, \bar{u})-\dot{\bar{x}}(t))\right] \mathrm{d} t .
\end{aligned}
$$

Since $\sum_{j=1}^{m} \bar{\lambda}^{i}(t)\left(g^{j}(t, \bar{x}, \bar{u})+\bar{x}(t)^{\mathrm{T}} \omega^{j}(t)\right)=0$, and $\bar{x}(t)^{\mathrm{T}} \hat{\omega}(t) \leq s\left(\bar{x}(t) \mid C^{j}\right)$, this yields,

$$
\begin{aligned}
\int_{I}\left[f(t, \bar{x}, \bar{u})+\bar{u}(t)^{\mathrm{T}} \hat{z}(t)\right] \mathrm{d} t & >\int_{I}\left[f(t, \bar{x}, \bar{u})+\bar{u}(t)^{\mathrm{T}} \bar{z}(t)\right] \mathrm{d} t \\
& \Rightarrow \int_{I} \bar{u}(t) \hat{z}(t) \mathrm{d} t>\int_{I} \bar{u}(t) \bar{z}(t) \mathrm{d} t \\
& \Rightarrow \int_{I} s(\bar{u}(t) \mid K) \mathrm{d} t>\int_{I} s(\bar{u}(t) \mid K) \mathrm{d} t .
\end{aligned}
$$

This is absurd. Hence $(\bar{x}, \bar{u})$ is an optimal solution of (CP).

\section{Converse Duality}

The problem (WCD) can be written as the follows:

Maximize: $\psi\left(x, \mu, z, \lambda^{1}, \cdots, \lambda^{m}, \omega^{1}, \cdots, \omega^{m}\right)$.

Subject to $x(a)=0, x(b)=0$

$$
\begin{gathered}
\theta^{1}\left(t, x(t) u(t), \lambda^{1}(t), \cdots, \lambda^{m}(t), \mu(t), \omega^{1}(t), \omega^{2}(t), \cdots, \omega^{m}(t)\right)=0, \quad t \in I . \\
\theta^{2}\left(t, x(t) u(t), z(t), \lambda^{1}(t), \cdots, \lambda^{m}(t), \mu(t), \omega^{1}(t), \omega^{2}(t), \cdots, \omega^{m}(t)\right)=0, \quad t \in I \\
z(t) \in K, \quad t \in I \\
\omega^{j}(t) \in C^{j}, \quad t \in I, \quad j=1,2, \cdots, m \\
\lambda(t) \geq 0, \quad t \in I, \quad j=1,2, \cdots, m
\end{gathered}
$$

where

$$
\begin{gathered}
\theta^{1}=\theta^{2}(t, x, u, z, \lambda, \mu)=f_{x}+\sum_{j=1}^{m} \lambda^{j}(t)\left(g_{x}^{j}+\omega^{j}(t)\right)+\mu(t)^{\mathrm{T}} h_{x}+\dot{\mu}(t), \\
\theta^{2}=\theta^{2}(t, x, u, z, \lambda, \mu)=f_{u}+z+\lambda^{\mathrm{T}} g_{x}+\mu^{\mathrm{T}} h_{u} .
\end{gathered}
$$

Consider $\theta^{1}\left(., x(),. u(),. \lambda(),. \omega_{2}^{1}(),. \cdots, \omega^{m}(),. \mu().\right)$ and $\theta^{2}(t, x(),. u(),. \lambda(),. z(.) \mu()$.$) as defining a map-$ pings $Q^{1}: X \times u \times \Lambda \times W^{1} \times W^{2} \times \cdots \times W^{m} \times V \rightarrow B^{1}$ and $Q^{2}: X \times u \times Z \times \Lambda \times V \rightarrow B^{2}$ respectively where $\Lambda$ is the space of piecewise smooth $\lambda, V$ is space of piecewise smooth $\mu, W^{j}$ is the space of piecewise of smooth $W^{j}, j=1,2, \cdots, m, B^{1}$ and $B^{2}$ are Banach spaces. $\theta^{1}=\left(x, u, \lambda, \mu, \omega^{1}, \omega^{2}, \cdots, \omega^{m}\right)$ and $\theta^{2}=(x, u, \lambda, \mu, z)$ with $\lambda=\left(\lambda^{1}, \cdots, \lambda^{m}\right)$. Here some restrictions are required on the equality constraints. For 
this it suffices that if the Fréchet derivatives

$$
Q^{\prime 1}=\left(\theta_{x}^{1}(), \theta_{u}^{1}(), \theta_{\lambda}^{1}(), \theta_{\mu}^{1}(), \theta_{\omega^{1}}^{1}(), \cdots, \theta_{\omega^{m}}^{1}()\right)
$$

and

$$
Q^{\prime 2}=\left(\theta_{x}^{2}(), \theta_{u}^{2}(), \theta_{\lambda}^{2}(), \theta_{\mu}^{2}(), \theta_{\omega^{1}}^{2}(), \cdots, \theta_{\omega^{m}}^{2}()\right),
$$

have weak * closed range.

Theorem 6. (Converse Duality): Assume

$\left(\mathbf{A}_{1}\right): f, g$ and $h$ are twice continuously differentiable.

$\left(\mathbf{A}_{2}\right):\left(x, u, \lambda, \mu, z, \omega^{1}, \cdots, \omega^{m}\right)$ is an optimal solution of (CP).

$\left(\mathbf{A}_{3}\right): Q^{\prime 1}$ and $Q^{\prime 2}$ have weak * closed range.

(A $\left.\mathbf{A}_{4}\right)$ : The matrix $\left(\begin{array}{ll}f_{x x}+\lambda^{\mathrm{T}}(t) g_{x x}+\mu(t)^{\mathrm{T}} h_{x x} & f_{u x}+\lambda^{\mathrm{T}}(t) g_{u x}+\mu^{\mathrm{T}}(t) h_{u x} \\ f_{u x}+\lambda^{\mathrm{T}}(t) g_{u x}+\mu(t)^{\mathrm{T}} h_{u x} & f_{u u}+\lambda^{\mathrm{T}}(t) g_{u u}+\mu(t)^{\mathrm{T}} h_{u u}\end{array}\right)$ is nonsingular.

Then $\bar{x}$ is an optimal solution of (CP) and the optimal values of (CP) and (WCD) are equal.

Proof: Since $\left(x, u, \lambda, \mu, z, \omega^{1}, \cdots, \omega^{m}\right)$ is an optimal solution of (WCD), by Theorem 1 there exists $\lambda_{0} \in R$, and piecewise smooth functions $\theta: I \rightarrow R^{m}, \phi: I \rightarrow R^{m}$, and $\eta: I \rightarrow R^{m}$ such that

$$
\begin{gathered}
\alpha\left\{f_{x}+\sum \lambda^{j}(t)\left(g_{x}^{j}+\omega^{j}(t)\right)+\mu(t)^{\mathrm{T}} h_{x}+\dot{u}(t)\right\}+\theta(t)^{\mathrm{T}}\left(f_{x x}+\lambda(t)^{\mathrm{T}} g_{x x}+\mu(t)^{\mathrm{T}} h_{x x}\right) \\
+\phi(t)\left(f_{u x}+\lambda^{\mathrm{T}}(t) g_{u x}+\mu(t)^{\mathrm{T}} h_{u x}\right)=0, \quad t \in I . \\
\alpha\left(f_{u}+z(t)+\lambda^{\mathrm{T}}(t) g_{u}+\mu^{\mathrm{T}}(t) h_{u}\right)+\theta(t)^{\mathrm{T}}\left(f_{x u}+\lambda^{\mathrm{T}}(t) g_{x u}+\bar{\mu}^{\mathrm{T}}(t) h_{x u}\right) \\
+\phi(t)^{\mathrm{T}}\left(f_{u u}+\lambda^{\mathrm{T}}(t) g_{u u}+\bar{\mu}^{\mathrm{T}}(t) h_{u u}\right)=0, \quad t \in I . \\
\alpha\left(g^{j}+x(t)^{\mathrm{T}} \omega^{j}(t)\right)+\theta(t)^{\mathrm{T}}\left(g_{x}^{j}+\omega^{j}(t)\right)+\phi(t) g_{u}^{j}+\eta^{j}(t)=0, \quad t \in I . \\
\alpha(h-\dot{x}(t))+\theta(t)^{\mathrm{T}} h_{x}+\phi^{\mathrm{T}}(t) h_{u}+\dot{\theta}(t)=0, \quad t \in I \\
\alpha \lambda^{j}(t)+x(t)+\theta(t)^{\mathrm{T}} \lambda^{j}(t) \in N_{c^{j}}\left(\omega^{j}(t)\right), \quad j=1,2, \cdots, m . \\
\alpha \mu(t)+\phi(t) \in N_{K}(z) \\
\eta^{\mathrm{T}}(t) \lambda(t)=0, \quad t \in I \\
(\alpha, \theta(t), \phi(t), \eta(t)) \geq 0, \quad t \in I \\
(\alpha, \theta(t), \phi(t), \eta(t)) \neq 0, \quad t \in I .
\end{gathered}
$$

Using (36) and (37) in (42) and (43) respectively, we obtain

$$
\begin{aligned}
& \theta(t)^{\mathrm{T}}\left(f_{x x}+\lambda(t)^{\mathrm{T}} g_{x x}+\mu(t)^{\mathrm{T}} h_{x x}\right)+\phi(t)\left(f_{u x}+\lambda^{\mathrm{T}}(t) g_{u x}+\mu(t)^{\mathrm{T}} h_{u x}\right)=0, \\
& \theta(t)^{\mathrm{T}}\left(f_{x u}+\lambda^{\mathrm{T}}(t) g_{x u}+\bar{\mu}^{\mathrm{T}}(t) h_{x u}\right)+\phi(t)^{\mathrm{T}}\left(f_{u u}+\lambda^{\mathrm{T}}(t) g_{u u}+\bar{\mu}^{\mathrm{T}}(t) h_{u u}\right)=0 .
\end{aligned}
$$

The equations can be combined in the matrix form as,

$$
\left(\begin{array}{ll}
f_{x x}+\lambda^{\mathrm{T}}(t) g_{x x}+\mu(t)^{\mathrm{T}} h_{x x} & f_{u x}+\lambda^{\mathrm{T}}(t) g_{u x}+\mu^{\mathrm{T}}(t) h_{u x} \\
f_{u x}+\lambda^{\mathrm{T}}(t) g_{u x}+\mu(t)^{\mathrm{T}} h_{u x} & f_{u u}+\lambda^{\mathrm{T}}(t) g_{u u}+\mu(t)^{\mathrm{T}} h_{u u}
\end{array}\right)\left(\begin{array}{c}
\theta(t) \\
\phi(t)
\end{array}\right)=0, \quad t \in I .
$$

This, due to the hypothesis $\left(\mathrm{A}_{4}\right)$ yields 


$$
\theta(t)=0=\phi(t), \quad t \in I
$$

Let $\alpha=0$, then (44) implies $\eta(t)=0, t \in I$, consequently we get $(\alpha, \eta(t), \theta(t), \phi(t)=0), t \in I$, contradicting (50), hence $\alpha>0$.

The relations (44) together with (48) and (45) respectively imply

$$
\begin{gathered}
g^{j}+x(t)^{\mathrm{T}} \omega^{j}(t)=\frac{-\eta^{j}(t)}{\alpha}, \quad t \in I \\
h^{\prime}-\dot{x}(t)=0, \quad t \in I .
\end{gathered}
$$

From (52) and $\lambda^{j}(t) \geq 0, t \in I$, we have

$$
\sum_{j=1}^{m} \lambda^{j}(t)\left(g^{j}+x(t)^{\mathrm{T}} \omega^{j}(t)\right)=0, \quad t \in I .
$$

From (53) along with $\mu(t) \geq 0, t \in I$, we obtain

$$
\mu(t)^{\mathrm{T}}(h-\dot{x}(t))=0, \quad t \in I .
$$

In view of (51) and definition of a normal cone (50) and (51), we have $x(t) \in N_{c^{j}}\left(\omega^{j}(t)\right), t \in I$, $j=1,2, \cdots, m$ and $u(t) \in N_{K}(z(t))$ implying

$$
x^{\mathrm{T}}(t) \omega^{j}(t)=s\left(x(t) \mid C^{j}\right), \quad j=1,2, \cdots, m
$$

and

$$
\bar{u}(t)^{\mathrm{T}} z(t)=s(u(t) \mid K)
$$

From (52) together with (56) and

$$
\eta^{j}(t) \geq 0, \quad t \in I, \quad i=1,2, \cdots, m
$$

imply

$$
g^{j}+s\left(x(t) \mid C^{j}\right) \leq 0, \quad t \in I .
$$

From (53) and (57), the feasibility of $\bar{x}$ for (CP) follows.

Consider

$$
\begin{aligned}
& \int_{I}\left(f(t, \bar{x}, \bar{u})+\bar{u}^{\mathrm{T}} z+\sum_{j=1}^{m} \bar{\lambda}^{j}\left(g^{j}(t, \bar{x}, \bar{u})+\bar{x}^{\mathrm{T}} \bar{\omega}^{j}\right)+\bar{\mu}(t)^{\mathrm{T}}(h(t, \bar{x}, \bar{u})-\bar{x})\right) \mathrm{d} t \\
& \quad=\int_{I}\left(f(t, \bar{x}, \bar{u})+\bar{u}(t)^{\mathrm{T}} \bar{z}(t)\right) \mathrm{d} t=\int_{I} f(t, \bar{x}, \bar{u})+s(\bar{u}(t) \mid K) \mathrm{d} t
\end{aligned}
$$

(by using (54), (55) and (56).

This implies that the values of objective functionals of the problem are equal. Consequently in view of the hypothesis of Theorem 1 it implies that $(\bar{x}, \bar{u})$ is an optimal solution of (CP).

\section{Special Cases}

Let for $t \in I . B(t)$ and $D^{j}(t),(j=1,2, \cdots, m)$ be positive semidefinite matrics and continuous on $I$. Then

$$
\left(u(t)^{\mathrm{T}} B(t) u(t)\right)^{\frac{1}{2}}=s(u(t) \mid K),
$$

where

$$
K=\left\{B(t) z(t) \mid z(t)^{\mathrm{T}} B(t) z(t) \leq 1, t \in I\right\}
$$


and

$$
\left(x(t)^{\mathrm{T}} D^{j}(t) x(t)\right)^{\frac{1}{2}}=s\left(x(t) \mid C^{j}\right), j=1,2, \cdots, m
$$

where

$$
C^{j}=\left\{D^{j}(t) \omega^{j}(t) \mid \omega^{j}(t)^{\mathrm{T}} D^{j}(t) \omega^{j}(t) \leq 1, t \in I\right\} .
$$

The control problems of the preceding section becomes as the following:

$\left(\mathbf{W C D}_{\mathbf{0}}\right)$ : Maximize

$$
\int_{I}\left[f(t, x, u)+u(t)^{\mathrm{T}} B(t) z(t)+\sum_{j=1}^{m} \lambda^{j}(t)\left(g^{j}(t, x, u)+x(t)^{\mathrm{T}} D^{j}(t) \omega^{j}(t)\right)+\mu(t)^{\mathrm{T}}(h(t, x, u)-\dot{x})\right] \mathrm{d} t .
$$

Subject to

$$
\begin{gathered}
x(a)=x(b) \\
f_{x}(t, x, u)+\sum_{j=1}^{m} \lambda^{j}(t)\left(g_{x}^{j}(t, x, u)+D^{j}(t) \omega^{j}(t)\right)+\mu(t)^{\mathrm{T}} h(t, x, u)=\dot{\mu}(t), \quad t \in I \\
f_{u}(t, x, u)+B(t) z(t)+\lambda(t)^{\mathrm{T}} g_{u}(t, x, u)+\mu(t)^{\mathrm{T}} h_{u}(t, x, u)=0, \quad t \in I \\
\lambda^{j}(t) \geq 0, \quad t \in I, \quad j=1,2, \cdots, m \\
z(t) \in K \text { and } \omega^{j}(t) \in C^{j}, j=1,2, \cdots, m .
\end{gathered}
$$

If $s\left(x \mid C^{j}\right), j=1,2, \cdots, m$ are deleted and $s(u \mid K)$ is replaced by $\left(u(t)^{\mathrm{T}} B(t) u(t)\right)^{\frac{1}{2}}$, the problem $\left(\mathrm{CP}_{0}\right)$ and $\left(\mathrm{WCD}_{0}\right)$ reduce to those studied by Chandra et al. [2].

\section{Related Nonlinear Programming Problems}

If the functions appearing (CP) and (WCD) are independent, of $t$ then these problems reduce to the following nonlinear programming problem with support functions not reported explicitly in the literature.

$\left(\mathbf{C P}_{\mathbf{0}}\right)$ : Minimize $f(x, u)+s(u \mid K)$

subject to $g^{j}(x, u)+s\left(x \mid C^{j}\right) \leq 0, j=1,2, \cdots, m$.

(WCD $\mathbf{W}_{0}$ : Maximize $f(x, u)+u^{\mathrm{T}} z+\sum_{j=1}^{m} \lambda^{j}\left(g^{j}(x, u)+\mu^{\mathrm{T}} \omega^{j}\right)+\mu^{\mathrm{T}} h(x, u)$

subject to $f_{x}(x, u)+\sum_{j=1}^{m} \lambda^{j}\left(g_{x}^{j}(x, u)+\omega^{j}\right)+\mu^{\mathrm{T}} h_{x}(x, u)=0$

$$
\begin{aligned}
& f_{u}(x, u)+z+\lambda^{\mathrm{T}} g_{u}(x, u)+\mu^{\mathrm{T}} h_{u}(x, u)=0 \\
& \lambda^{j} \geq 0, \quad j=1,2, \cdots, m \\
& z \in K, \omega^{j} \in C^{j}, j=1,2, \cdots, m .
\end{aligned}
$$

If $f(x, u)$ and $s(u \mid K)$ are replaced by $f(x)$ and $s(x \mid K)$ respectively, the above problem reduce to the following problem studied by Husain et al. [12].

(NP): Minimize $f(x)+s(x \mid K)$.

Subject to $g^{j}(x)+s\left(x \mid C^{j}\right) \leq 0, j=1,2, \cdots, m$.

(WNP 1 ): Maximize $f(x)+x^{\mathrm{T}} z+\sum_{j=1}^{m} \lambda^{j}\left(g^{j}(x)+x^{\mathrm{T}} \omega^{j}\right)+\mu^{\mathrm{T}} h(x)$. 


$$
\begin{array}{ll}
\text { Subject to } & f_{x}(x)+z+\sum_{j=1}^{m} \lambda^{j}\left(g_{x}^{j}(x)+\omega^{j}\right)+\mu^{\mathrm{T}} h_{x}=0 \\
& \lambda^{j} \geq 0, \quad j=1,2, \cdots, m \\
& z \in K, \omega^{j} \in C^{j}, j=1,2, \cdots, m .
\end{array}
$$

\section{Conclusion}

Fritz John and Karush-Kuhn-Tucker type necessary optimality conditions for class of nondifferentiable control problems are derived. As an application of Karush-Kuhn-Tucker type necessary optimality conditions, Wolfe type dual is formulated and various duality theorems under generalized convexity conditions are proved. The linkage between our duality results and those of a nonlinear programming problem with support functions is indicated.

\section{References}

[1] Mond, B. and Hanson, M.A. (1968) Duality for Control Problems. SIAM Journal on Control and Optimization, 6, 114-120.

[2] Chandra, S., Craven, B.D. and Husain, I. (1988) A Class of Nondifferentiable Control Problem. Journal of Optimization Theory and Applications, 56, 227-243. http://dx.doi.org/10.1007/BF00939409

[3] Hanson, M.A. (1968) Bounds for Functionally Convex Optimal Control Problems. Journal of Mathematical Analysis and Applications, 8, 84-89. http://dx.doi.org/10.1016/0022-247X(64)90086-1

[4] Pearson, J.D. (1965) Reciprocality and Duality in Control Problems. Journal of Mathematical Analysis and Applications, 10, 383-408. http://dx.doi.org/10.1016/0022-247X(65)90134-4

[5] Ringlee, R.J. (1965) Bounds for Convex Variational Programming Problems Arising in Power System, Scheduling and Control. IEEE Transaction on Automatic Control, 10, 28-35. http://dx.doi.org/10.1109/TAC.1965.1098077

[6] Clarke, F.H. (1983) Optimization and Nonsmooth Analysis. Wiley, New York.

[7] Demyanov, V.F. and Dixon, L.C.W. (1986) Quasidifferential Calculus. North-Holland, Amsterdam. http://dx.doi.org/10.1007/BFb0121132

[8] Mond, B. and Schechter, M. (1996) Nondifferentiable Symmetric Duality. Bulletin of the Australian Mathematical Society, 53, 177-188. http://dx.doi.org/10.1017/S0004972700016890

[9] Craven, B.D. and Mond, B. (1979) Langrangean Conditions for Quasi-Differentiable Optimization. Proceedings of the 9th International Conference on Mathematical Programming, Akademiai Kiado, Budapest and North-Holand, Amstedam.

[10] Kreindler, F. (1966) Reciprocal Optimal Control Problems. Journal of Mathematical Analysis and Applications, 14, 141-152. http://dx.doi.org/10.1016/0022-247X(66)90067-9

[11] Husain, I. and Jabeen, Z. (2008) Continuous Programming Containing Support Functions. Journal of Applied Mathematics and Informatics, 26, 75-106.

[12] Husain, I., Abha and Jabeen, Z. (2002) On Nonlinear Programming with Support Functions. Journal of Applied Mathematics and Computing, 10, 83-99. 
Scientific Research Publishing (SCIRP) is one of the largest Open Access journal publishers. It is currently publishing more than 200 open access, online, peer-reviewed journals covering a wide range of academic disciplines. SCIRP serves the worldwide academic communities and contributes to the progress and application of science with its publication.

Other selected journals from SCIRP are listed as below. Submit your manuscript to us via either submit@scirp.org or Online Submission Portal.
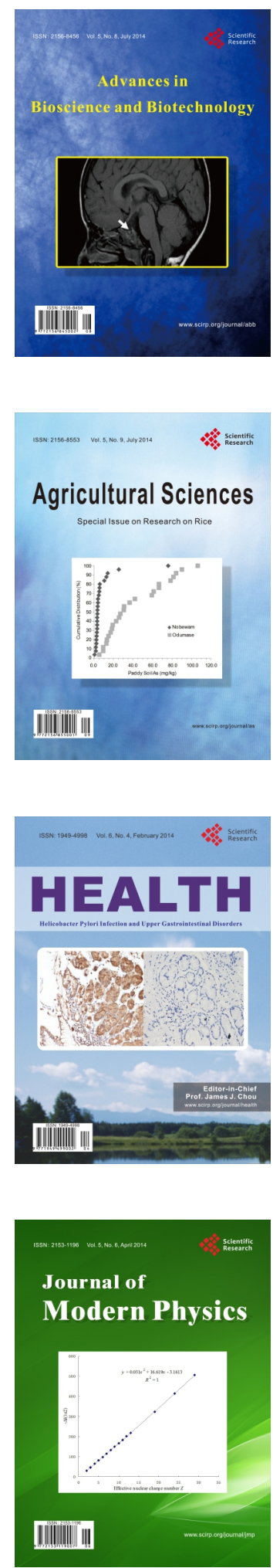
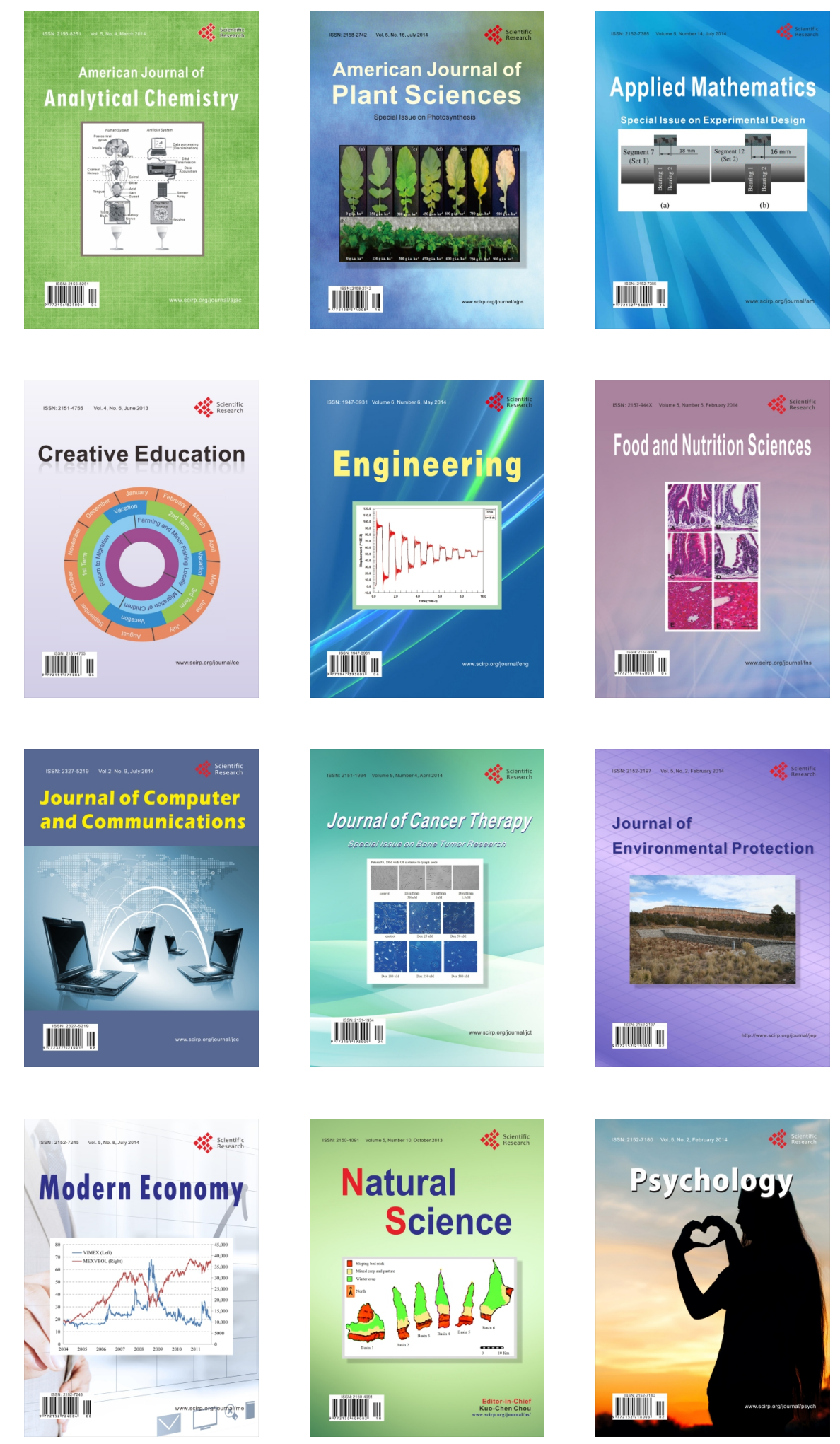